\title{
Gas Dynamic Simulation of the Star-Planet Interaction using a Binary Star Model
}

\author{
D. E. Ionov, D. V. Bisikalo, P. V. Kaygorodov, V. I. Shematovich \\ Institute of Astronomy of the Russian Acad. of Sci., Pyatnitskaya 48, Moscow, Russia
}

\begin{abstract}
We have performed numerical simulations of the interaction between a "hot Jupiter" planet and gas of the stellar wind using a numerical code developed for investigations of binary stars. With this code, we have modeled the structure of the gaseous flow in the system HD 209458. The results have been used to explain observations of this system performed with the COS instrument on-board the HST.
\end{abstract}

Keywords. planetary systems, binaries, hydrodynamics

The observations of a "hot Jupiter" planet HD 209458b were carried out using the COS spectrograph mounted aboard HST (Linsky 2010). The results showed that the investigated spectral absorption lines (of CII, Si III) obtained as the difference of the stellar spectra in the transition and out of it have a non-trivial double-peaked shape. The distance between the peaks is about $20 \mathrm{~km} / \mathrm{s}$ and for the carbon line it is clearly seen that the peaks are asymmetric.

To reveal physical processes that can lead to formation of such spectral lines, we performed gas dynamic simulations of the interaction between a planet and stellar wind. Since the planet is pretty close to the host star $\left(10.1 R_{\text {sun }}\right)$, the orbital velocity of the planet is so high $(\mathrm{V}=143 \mathrm{~km} / \mathrm{s})$ that even in the case of a hot stellar wind with temperatures around $10^{5} \mathrm{~K}$ the motion of the planet is supersonic. It is known that if a gravitating body or a body with an atmosphere moves with a supersonic velocity, a bowshock must occur. The matter of the stellar wind mixes with the matter of the atmosphere and forms two streams moving in different directions from the head-on collision point. This motion can lead to occurrence of two peaks in the observed spectral lines.

The model we have used for our numerical experiments is similar to that described in (Bisikalo et al. 2003a). The flow structure in this model is described by a system of 3D equations of gravitational gas dynamics including non-adiabatic processes of the radiative heating and cooling. To obtain the numerical solution of this system, we have used the Roe-Osher method (Boyarchuk et al. 2002; Roe, 1986; Chakravarthy \& Osher, 1985; Bisikalo et al. 2003b) adapted to perform simulations using multiprocessor computers. We carried out our calculations in a cylindrical coordinate system with the origin at the center of mass of the planet. The size of the computational domain has been limited to 5 planet radii. The stellar wind was simulated through setting a constant inflow on the outer boundary of the computational domain. The matter of the stellar wind is considered as a neutral monatomic gas whose parameters are typical for the Solar wind, i.e.: $\rho=$ $1,4 \cdot 10^{4} \mathrm{~cm}^{-3}, T=7,3 \cdot 10^{5} K, V=100 \mathrm{~km} / \mathrm{s}$ (Withbroe 1988). The planet atmosphere is set to isothermal, with $T_{p l}=5000 \mathrm{~K}$. Its number density has been determined using the barometric formula. The number density at the radius $R_{p l}=1.4 R_{J u p}$ has been set as $2 \cdot 10^{10} \mathrm{~cm}^{-3}$. 
The shape of the line is determined by projections of the velocities of matter behind the bow shock wave onto the line of sight. In Fig. 1 (right panel), we show the synthetic absorption line profile calculated using our gas dynamic results. It is seen that the line has two clearly distinguishable asymmetric peaks. The synthetic line profile has the same features as the observed one. Parameters of the line (peak position, their relative intensity and even their number) strongly depend on the accepted parameters of the modeled stellar wind and atmosphere, and can vary in a wide range.
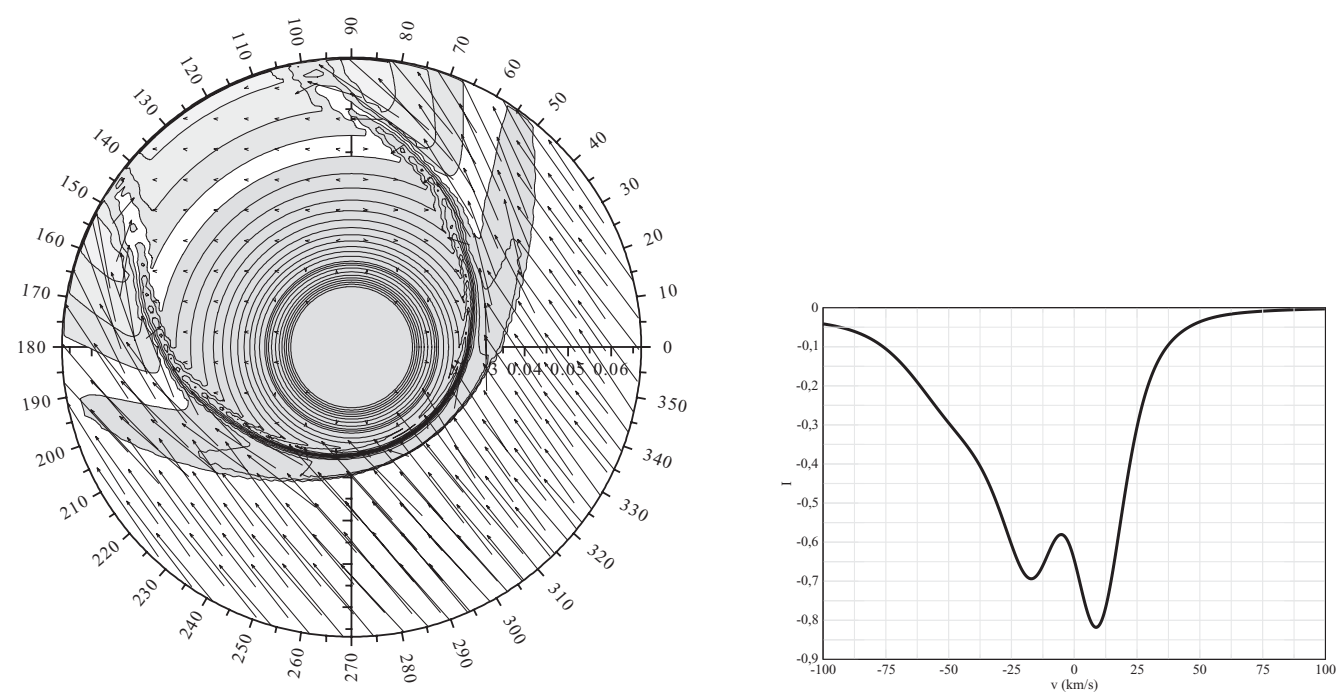

Figure 1. Density distribution and velocity vectors in the equatorial plane (left panel) and synthetic spectral line profile(right panel).

The presented model allows us to conclude that when analyzing observational properties of the atmosphere of a planet, one must take into account gas dynamical processes caused by the interaction of the atmosphere and stellar wind. It is important to note that the considered model allows one to explain the existence of absorption lines of ions having high ionization potentials.

\section{Acknowledgements}

This work was supported by the Basic Research Program of the Presidium of the Russian Academy of Sciences, Russian Foundation for Basic Research (projects 09-02-00064, 09-02-00993, 11-02-00076, 11-02-01248), and the Federal Targeted Program "Science and Science Education for Innovation in Russia 2009-2013."

\section{References}

Bisikalo, D. V., Boyarchuk, A. A., Kaigorodov, P. V., \& Kuznetsov, O. A. 2003, Astronomy Reports, 47, 10, 809

Bisikalo, D. V., Boyarchuk, A. A., Kaigorodov, P. V., \& Kuznetsov, O. A. 2003, Matematicheskoye modelirovanie: Problemi i rezultati, 71

Boyarchuk, A. A., Bisikalo, D. V., Kuznetsov, O. A., \& Chechetkin, V. M. 2002, Mass Transfer in Close Binary Stars (London: Taylor \& Francis)

Chakravarth, S. R. \& Osher, S. 1985, Proceedings of the $23^{r d}$ Aerospace Sci. Meeting, p. 363

Linsky, J. L., Hao, Y., France, K., Froning, C. S., Green, J. C., Stocke, J. T., \& Osterman, S. N. 2010, ApJ, 717, 1291

Roe, P. L. 1986, Ann. Rev. Fluid Mech., 18, 337

Withbroe, G. L. 1988, ApJ, 325, 442 